appears that such abnormalities are mostly observed during the first two years of TKI therapy. While there is a significant risk of a second myeloid malignancy in patients with -7 CCA/Ph; less than half of these patients will develop MDS/AML. The aggregate data provide evidence in support of the commonly held view that preemptive therapeutic strategies are not justified in all patients with detectable -7 CCA/Ph: Nevertheless, once a diagnosis of AML is confirmed in these patients, intensive treatment strategies, including allogeneic BM transplantation, are ineffective in most patients. One may speculate on the role of TKI in the mechanism of MDS development and the presence of $-7 / \operatorname{del}(7 q) \mathrm{CCA} / \mathrm{Ph}$ abnormalities. The mutagenic effect of TKI on hematopoietic stem cells is not yet fully understood. However, it has been reported that a gastrointestinal stromal tumor patient developed MDS with monosomy 7 during imatinib treatment, suggesting that imatinib plays a direct role in causing MDS. ${ }^{12}$

The routine monitoring of CML patients is currently molecular assessment of the response. However, cytogenetic analysis is still relevant and should be performed with a BM smear certainly in cases of cytopenia during TKI therapy. Signs of dysplasia with -7 CCA/Ph cells should be considered as a red signal and a switch to alternative treatment be discussed.

\section{References}

1. Guilhot F. Cytogenetics in CML: more important than you think. Blood. 2016;127(22):2661-2662.
2. Wang W, Cortes JE, Tang G, et al. Risk stratification of chromosomal abnormalities in chronic myelogenous leukemia in the era of tyrosine kinase inhibitor therapy. Blood. 2016;127(22):2742-2750.

3. Baccarani M, Deininger MW, Rosti G, et al. European LeukemiaNet recommendations for the management of chronic myeloid leukemia: 2013. Blood. 2013;122(6):872-884.

4. National Comprehensive Cancer Network (NCCN). Chronic myeloid leukemia (version 1.2019). Available at: https://www.nccn.org.

5. Groves MJ, Sales M, Baker L, Griffiths M, Pratt N, Tauro S. Factors influencing a second myeloid malignancy in patients with Philadelphia-negative -7 or $\operatorname{del}(7 q)$ clones during tyrosine kinase inhibitor therapy for chronic myeloid leukemia. Cancer Genet. 2011;204(1):39-44

6. Wasilewska EM, Panasiuk B, Gniot M, et al. Clonal chromosomal aberrations in Philadelphia negative cells such as monosomy 7 and trisomy 8 may persist for years with no impact on the long-term outcome in patients with chronic myeloid leukemia. Cancer Genet. 2017;216-217:1-9.

7. Issa GC, Kantarjian HM, Gonzalez GN, et al. Clonal chromosomal abnormalities appearing in Philadelphia chromosome-negative metaphases during CML treatment. Blood. 2017;130(19):2084-2091.

8. Bidet A, Dulucq S, Smol T, et al. Poor prognosis of chromosome 7 clonal aberrations in Philadelphia-negative metaphases and relevance of potential underlying myelodysplastic features in chronic myeloid leukaemia. Hematologica. 2019;104(6):1150-1155.

9. Gambacorti-Passeri C, Giudici G, le Coutre P, et al. Non-random chromosomal abnormalities in Ph-negative bone marrow (BM) cells from CML patients achieving major cytogenetic responses (MCR) with STI571 (GleevecTM). Blood. 2001;98:257b (Abstr. 4762).

10. Andersen MK, Pedersen-Bjergaard J, Kjeldsen L, Dufva IH, BrøndumNielsen K. Clonal Ph-negative hematopoiesis in CML after therapy with imatinib mesylate is frequently characterized by trisomy 8. Leukemia. 2002;16(7):1390-1393.

11. Karimata K, Masuko M, Ushiki T, et al. Myelodysplastic Syndrome with Ph Negative Monosomy 7 Chromosome following Transient Bone Marrow Dysplasia during Imatinib Treatment for Chronic Myeloid Leukemia. Intern Med. 2011;50(5):481-485.

12. Pitini V, Arrigo C, Sauta MG, Altavilla G. Myelodysplastic syndrome appearing during imatinib mesylate therapy in a patient with GIST. Leuk Res. 2009;33(9):143-144.

\title{
Another piece of the puzzle added to understand $t(4 ; 11)$ leukemia better
}

\section{Rolf Marschalek}

Institute of Pharmaceutical Biology / Diagnostic Center of Acute Leukemia, University of Frankfurt, Frankfurt/Main, Germany; E-mail: ROLF MARSCHALEK - Rolf.Marschalek@em.uni-frankfurt.de

doi:10.3324/haematol.2018.213397

$\mathrm{T}$ he story about $t(4 ; 11)$ leukemia, involving the MLL/KMT2A gene from chromosome 11q23.3 and the AF4/AFF1 gene from chromosome 4q21, is still a mystery. The study by Agraz-Doblas et al., published in this issue of Haematologica, adds some new and important information regarding the mysterious pathomechanism. ${ }^{1}$ Agraz-Doblas et al. showed, for the first time, that the therapeutic outlook of patients with expression of both reciprocal MLL fusions, MLL-AF4 and AF4-MLL, is promising, but only $50 \%$ of the investigated patients seem to have this favorable condition; patients expressing only the MLL-AF4 allele have an event-free survival of $10 \%$ and an overall survival of $30 \%$. Moreover, only leukemic cells expressing both fusion alleles display the typical HOXA signature.

The fact that $\mathrm{t}(4 ; 11)$ patients can be divided into two subgroups on the basis of HOXA transcription was first recognized by Trentin et al. in $2009,{ }^{2}$ and later confirmed by Stam et al. and Kang et al. in 2010 and 2012, respectively. ${ }^{3,4}$ The missing HOXA transcription was correlated with overexpression of either IRX1 or IRX22,4 and a 3-fold higher relapse rate ${ }^{3,4}$ Experimental overexpression of IRX1 revealed an interesting mechanism because it resulted in EGR1-3 expression. ${ }^{5}$ EGR1 and EGR2 both control the P21 $1^{\text {CIP }}$ gene and, thus, shut down the cell cycle and may even induce cellular quiescence, a known mechanism of resistance to treatment. CDK6 counteracts the actions of EGR proteins. ${ }^{6}$ The second mechanism involves the IRX proteins, which are able to turn on HOXB4, a known stem cell marker of hematopoietic cells that activates factors such as TAL1, GATA factors, TGFB1, etc. Thus, expression of MLL-AF4 alone - with upregulated IRX proteins but without HOXA expression - may provoke treatment resistance or a stem cell-like mechanism which is 
not possible when AF4-MLL is present. This could be a rational explanation for the observed clinical behavior of both groups of patients.

Another explanation could lie in the recent findings from Yokoyama's laboratory. ${ }^{7,8}$ Okuda et al. elegantly showed that one of the functions of the pSer domain of the AF4 protein ${ }^{9}$ - which is fused to the $\mathrm{N}$-terminus of MLL in the MLL-AF4 fusion protein - is recruitment of the SL1 complex. The SL1 complex is usually bound to RNA Pol I, which is present in the nucleolus and required for the transcription of rRNA genes. SL1 is artificially recruited to MLL-AF4, but not to native MLL or AF4 complexes. ${ }^{10}$ This powers up MLL-AF4 leading to a strong increase in gene transcription. The simple presence of the MLLAF4 fusion protein causes a condition of severe stress, because it compromises protein biosynthesis, and cells may therefore easily display a phenotype of growth arrest or senescence. ${ }^{11}$ This is probably one of the reasons why it is so complicated to generate a true MLL-AF4 mouse model, and why so many laboratories have failed so far. With the exception of a recent study in which a hybrid between human MLL and mouse Af4 was used, ${ }^{12}$ no-one had been able to develop a satisfactory model with only the human MLL-AF4 fusion. Since the sequences of the pSer domains of human and mouse AF4 differ slightly, it is very plausible that the human/mouse chimeric MLL-Af4 is unable to attract the SL1 component and thus does not impair protein biosynthesis. This needs to be tested in future experiments.

So, what is the precise role of AF4-MLL? AF4-MLL has been shown to strongly enhance gene transcription by overwriting the transcriptional elongation control. ${ }^{10,13}$ This massive increase in gene transcription (3- to 4-fold more mRNA) may help its molecular counterpart, MLLAF4, to set the programming of its target genes, even under conditions of nucleolar stress (see Figure 1). In addition, we have shown by ATAC sequencing in two independent cell lines that AF4-MLL strongly activates chromatin in a very short time frame (unpublished data from our laboratory). The expression of AF4-MLL for $48 \mathrm{~h}$ was sufficient to open up the chromatin of all chromosomes apart from the centromeric regions and to massively increase gene transcription. Thus, the presence of AF4MLL would allow the expression of any gene of interest, and increases the plasticity of the tumor cells. According to the data shown by Agraz-Doblas et al. this results in

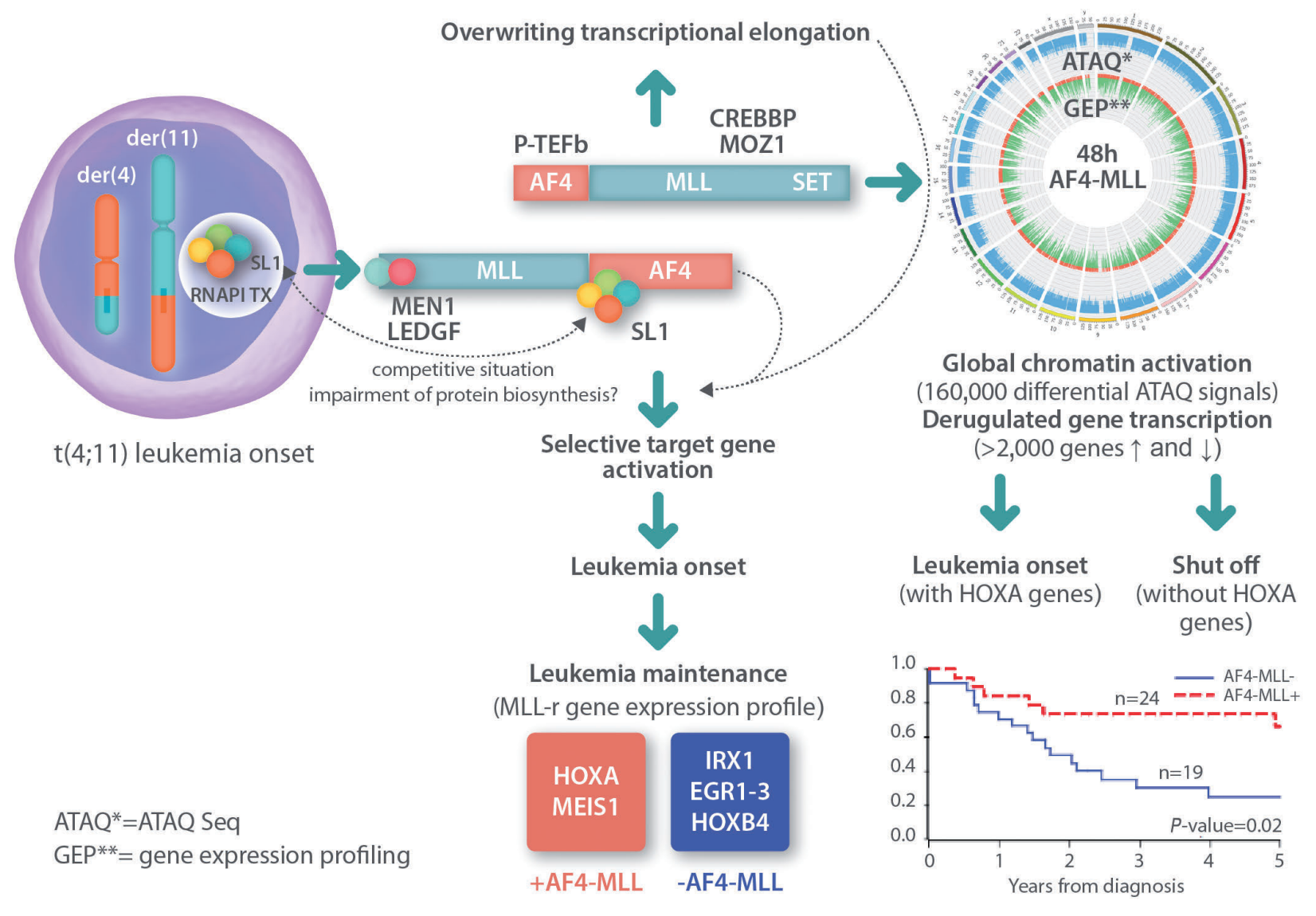

Figure 1. Proposed fuctions of both $\mathrm{t}(4 ; 11)$ fusion proteins. MLL-AF4 binds to Menin1/LEDGF and SL1 to target gene promotors and strongly activate gene transcription, while AF4-MLL overwrites transcriptional elongation control and strongly activates chromatin within a very short time window. Patients who express both fusions diplay HOXA gene signatures and have a better outcome, while patients who either do not express AF4-MLL or actively repress it, usually activate the homeobox proteins IRX1/2. Expression of both proteins has been correlated with a worse outcome (IRX1/2 strongly activate EGR1-3 and HOXB4). 
cells that are more vulnerable to chemotherapy because the presence of AF4-MLL was associated with a much better treatment outcome. ${ }^{1}$

Lastly, expression of AF4-MLL alone was shown to be necessary and sufficient to cause acute leukemia. ${ }^{14}$ Our group used a low expression retroviral vector backbone (PIDE vector) to express MLL-AF4, AF4-MLL or both in LSK cells purified from C57BL/6 mice. Empty vector or MLL-AF4 alone did not result in the development of leukemia, while AF4-MLL or the expression of both fusion genes resulted in full-blown pro-B-acute lymphoblastic leukemia and mixed-lineage leukemia. The latency was 9 months and the penetrance was only 35\%. However, this could be attributed to a low infection rate with about 1/1,000 cells for the AF4-MLL and 1/10,000 cells for the MLL-AF4 construct because these constructs were oversized for in vitro packaging $(11.3 \mathrm{~kb}$ for MLLAF4 and $13.3 \mathrm{~kb}$ for AF4-MLL). Therefore, an estimated 200 cells in 200,000 non-tranduced cells were transplanted into primary mice, which nevertheless caused a disease outbreak (MLL-AF4 with only 20 cells did not work). It is noteworthy that all "leukemic cells" subsequently tested positive for the transcription and integrity of the appropriate transgenes, while the investigated white blood cells of mice who did not develop leukemia remained negative in reverse transcriptase and genomic polymerase chain reaction experiments. This indicates that the leukemia-negative mice either never received or lost the cells carrying the corresponding transgene (negative selection of MLL-AF4 alone).

We, therefore, assume that, in humans, AF4-MLL and MLL-AF4 are both necessary, but AF4-MLL could presumably be shut off after "preparing the ground" for MLL-AF4, and that this process of shutting down AF4MLL makes the leukemic disease even more aggressive (positive selection). This explains in part the molecular situation diagnosed in human patients with leukemia, regardless of whether they are infants or adults. It would be of interest to compare primary diagnostic material with relapsed material from the same patient, and determine whether AF4-MLL expression is lost in the relapse, in order to have another argument in favor of the above mentioned hypothesis.
The study by Agraz-Doblas et al. adds another, important piece to the puzzle of the molecular mechanism of $\mathrm{t}(4 ; 11)$ leukemia. ${ }^{1}$ It is to be hoped that the precise mechanism of this disease can be understood soon, because the full picture is needed in order to develop new drugs that can really help patients with $t(4 ; 11)$ leukemia.

\section{References}

1. Agraz-Doblas A, Bueno C,2 Bashford-Rogers R, et al. Unraveling the cellular origin and clinical prognostic markers of infant B-cell acute lymphoblastic leukemia using genome-wide analysis. Haematologica 2019;104(6):1176-1188.

2. Trentin L, Giordan M, Dingermann T, et al. Two independent gene signatures in pediatric $t(4 ; 11)$ acute lymphoblastic leukemia patients. Eur J Haematol. 2009;83(5):406-419.

3. Stam RW, Schneider P, Hagelstein JA, et al. Gene expression profiling-based dissection of MLL translocated and MLL germline acute lymphoblastic leukemia in infants. Blood. 2010;115(14):2835-2844.

4. Kang H, Wilson CS, Harvey RC, et al. Gene expression profiles predictive of outcome and age in infant acute lymphoblastic leukemia: a Children's Oncology Group study. Blood. 2012;119(8):1872-1881.

5. Kühn A, Löscher D, Marschalek R. The IRX1/HOXA connection: insights into a novel t(4;11)- specific cancer mechanism. Oncotarget. 2016;7(23):35341-35352.

6. Guzman ML. CDK6 is a regulator of stem cells "Egr" to wake up. Blood. 2015;125(1):7-9

7. Okuda H, Kanai A, Ito S, Matsui H, Yokoyama A. AF4 uses the SL1 components of RNAP1 machinery to initiate MLL fusion- and AEPdependent transcription. Nat Commun. 2015;6:8869.

8. Okuda H, Takahashi S, Takaori-Kondo A, Yokoyama A. TBP loading by AF4 through SL1 is the major rate-limiting step in MLL fusiondependent transcription. Cell Cycle. 2016;15(20):2712-2722.

9. Nilson I, Reichel M, Ennas MG, et al. Exon/intron structure of the human AF-4 gene, a member of the AF-4/LAF-4/FMR-2 gene family coding for a nuclear protein with structural alterations in acute leukaemia. Br J Haematol. 1997;98(1):157-169.

10. Benedikt A, Baltruschat S, Scholz B, et al. The leukemogenic AF4MLL fusion protein causes P-TEFb kinase activation and altered epigenetic signatures. Leukemia. 2011;25(1):135-144.

11. Caslini C, Serna A, Rossi V, Introna M, Biondi A. Modulation of cell cycle by graded expression of MLL-AF4 fusion oncoprotein. Leukemia. 2004;18(6):1064-1071

12. Lin S, Luo RT, Ptasinska A, et al. Instructive role of MLL-fusion proteins revealed by a model of $\mathrm{t}(4 ; 11)$ pro-B acute lymphoblastic leukemia. Cancer Cell. 2016;30(5):737-749.

13. Mück F, Bracharz S, Marschalek R. DDX6 transfers P-TEFb kinase to the AF4/AF4N (AFF1) super elongation complex. Am J Blood Res. 2016;6(3):28-45.

14. Bursen A, Schwabe K, Rüster B, et al. The AF4-MLL fusion protein is capable of inducing ALL in mice without requirement of MLL-AF4. Blood. 2010;115(17):3570-3579. 